\title{
Spontaneous Periodic Hypothermia in the Elderly: A Rare or Under-Recognised Syndrome
}

\author{
Hassene Attout, Sofia Amichi, Youcef Belkheir
}

Internal Medicine Unit, General Hospital, Ales, France

Received: 06/07/2020

Accepted: $13 / 07 / 2020$

Published: $28 / 09 / 2020$

How to cite this article: Attout H, Amichi S, Belkheir Y. Spontaneous periodic hypotermia in the elderly: a rare or under-recognised syndrome. EJCRIM 2020;7: doi:10.12890/2020_001874.

Conflicts of Interests: The Authors declare that there are no competing interests.

This article is licensed under a Commons Attribution Non-Commercial 4.0 License

\section{ABSTRACT}

Spontaneous periodic hypothermia is a rare syndrome presenting with recurrent, centrally mediated hypothermia without an identifiable systemic cause or brain lesion. The case of an 88-year-old woman with recurrent hypothermia is reported. Despite intensive investigation, no other manifestations of hypothalamic or autonomic dysfunction were found. No corpus callosum lesion was seen on MRI. The patient was successfully treated with clomipramine chlorhydrate.

\section{LEARNING POINTS}

- Periodic hypothermia in the elderly is a rare and possibly under-recognised syndrome.

- The causes of the syndrome remain unknown.

- Pharmacological trials have only been modestly successful.

KEYWORDS

Periodic hypothermia, Shapiro's syndrome, clomipramine

\section{INTRODUCTION}

Spontaneous periodic hypothermia (SPH), defined as a core temperature below $35^{\circ} \mathrm{C}$, is rare and occurs in association with a range of brain pathologies, including congenital brain disease, and acquired traumatic, ischaemic, neoplastic or inflammatory mechanisms involving the hypothalamus. In 1969, Shapiro et al. described the first two cases of SPH associated with agenesis of the corpus callosum ${ }^{[1]}$. Since then, numerous cases of idiopathic recurrent hypothermia have been described without corpus callosum agenesis. Structural, epileptiform or biochemical abnormalities have been suggested as possible causes. However, the aetiology of SPH has not yet been elucidated. Pharmacological trials to date have been only modestly successful. Anticonvulsant agents, clonidine, clomipramine and cyproheptadine appear the most likely successful treatments ${ }^{[2,3]}$.

\section{CASE DESCRIPTION}

An 88-year-old woman presented to the emergency department with repeated episodes of hypothermia and confusion over a 4-year period. Her medical history included hypertension, hypothyroidism and colonic diverticular disease. Her regular medications were aspirin, furosemide and levothyroxine. The patient was a non-smoker and did not consume alcohol. There was no history of abnormal behaviour or seizures. Her family history was unremarkable. 
The patient first's episode of hypothermia occurred in April 2016. On each of her five admissions, her peripheral body temperature recorded on arrival ranged from $30^{\circ} \mathrm{C}$ to $34^{\circ} \mathrm{C}$.

During each episode, the patient presented with confusion, dysarthria and mild ataxia. No hyperhidrosis was noted. There was no seasonal or diurnal variation. On each occasion, the hypothermia, confusion and ataxia resolved within 3-5 days with only supportive therapy. Between episodes of hypothermia, the patient returned to normal function without complaints despite her advanced age.

Septic screening tests were consistently negative. Investigation of anterior pituitary, thyroid and adrenal cortical function was normal at baseline and during hypothermia events. All biochemistry tests were within normal limits. CSF study was also normal. No antibodies against neuronal cell-surface antigens were found. Other investigations, including thermoregulatory sweat testing and autonomic reflex screening, were unremarkable

Brain MRI was carried out three times and showed only mild atrophy. The corpus callosum, hypothalamus and pituitary were normal. An EEG was performed during each episode without findings of seizures or focal abnormalities. A diagnosis of SPH was made.

The patient was treated with lamotrigine and levetiracetam for 12 months without any response. Finally, in February 2019, oral clomipramine $25 \mathrm{mg}$ per day was initiated and the patient remains asymptomatic to date.

\section{DISCUSSION}

Thermoregulation maintains the temperature of the human body at $37^{\circ} \mathrm{C}$ by a complex mechanism. Induced or accidental hypothermia may occur in multiple clinical settings and may be caused by metabolic disorders, endocrine disorders, malnutrition, drugs, dermal dysfunction, sepsis and CNS dysfunction ${ }^{[2,4-6]}$. The results of a review of the national French pharmacovigilance database showed that nearly a quarter (153/614) of cases of drug-related hypothermia were attributed to psychotropic drugs, mainly neuroleptics ${ }^{[4]}$.

Very rarely, periodic hypothermia has been observed with agenesis of the corpus callosum (Shapiro's syndrome), with CNS abnormalities affecting structures related to thermoregulation, and without an associated systemic disease or obvious brain lesion. Prodromal sweating was a feature of most cases ${ }^{[1-3]}$.

Shapiro's syndrome is characterized by spontaneous recurrent episodes of hypothermia, hyperhidrosis and corpus callosum (CC) agenesis. Less than 80 cases have been reported to date and the pathogenic mechanism as well as the prognosis of this syndrome is still debated. It was suggested that episodes of hypothermia were due to a paroxystic reset of the hypothalamic thermostat with a lower temperature set point ${ }^{[1]}$.

Our case is consistent with previously reported cases of SPH. In those cases, the periodicity of hypothermia attacks ranged from hours to years and episodes lasted from hours to weeks ${ }^{[2,3]}$. In our case, Shapiro's syndrome was excluded due to the age of patient and absence of corpus callosum agenesis. A degenerative or autoimmune process was also excluded.

There is general consensus among authors that an altered central thermoregulatory mechanism is the determining factor for the fluctuating body temperatures in both Shapiro's syndrome and SPH. Some authors have postulated an epileptic basis for SPH on the basis of EEG changes and some responsiveness to anticonvulsant therapy. However, others have noted that several antiepileptic drug trials have failed, and argue that the EEG changes may be secondary to hypothermia ${ }^{[2,7]}$. Rodrigues et al. found a consistent abnormality in cerebral neurotransmitter levels in patients with SPH without corpus callosum agenesis ${ }^{[8]}$. Recently, Tambasco et al. highlighted that agenesis of the corpus callosum was seen in only $40 \%$ of cases reported in the literature ${ }^{[9]}$.

Drug therapy with clonidine, oxybutynin, carbamazepine, pizotifen, clomipramine and chlorpromazine has been successful in rare cases ${ }^{[4,7]}$. In our patient, treatment with anticonvulsants (levetiracetam, lamotrigine) was tried without any response. Eventually our patient was treated with low-dose clomipramine $(25 \mathrm{mg} /$ day) and after 18 months, she remains free from hypothermia attacks.

\section{REFERENCES}

1. Shapiro WR, Williams GH, Plum F. Spontaneous recurrent hypothermia accompanying agenesis of the corpus callosum. Brain 1969;92:423-436.

2. Blondin NA. Diagnosis and management of periodic hypothermia. Neurol Clin Pract 2014;4(1):26-32.

3. Kloos RT. Spontaneous periodic hypothermia. Medicine (Baltimore) 1995;74(5):268-280.

4. Bosacki C, Hausfater P, Koenig M, Serratrice J, Piette AM, Cathebras P. Spontaneous hypothermia: a series of ten cases, place of Shapiro's syndrome. Rev Med Interne 2005;26(8):615-623.

5. Mayer U, Aigner JM, Klein HE. Hypothermia caused by neuroleptics: 2 case reports and review of the literature. Nervenarzt 1994;65(7):488-491.

6. Hemelsoet DM, De Bleecker JL. Post-traumatic spontaneous recurrent hypothermia: a variant of Shapiro's syndrome. Eur J Neurol 2007;14(2):224-227.

7. Walker BR, Anderson JA, Edwards CR. Clonidine therapy for Shapiro's syndrome. Q J Med 1992;82(299):235-245.

8. Rodrigues MM, Lin J, Arita JH, De Castro Neto EF, Scerni DA, Cavalheiro EA, et al. Spontaneous periodic hypothermia and hyperhidrosis: a possible novel cerebral neurotransmitter disorder. Dev Med Child Neurol 2011;53:378-380.

9. Tambasco N, Belcastro V, Prontera P, Nigro P, Donti E, Rossi A, et al. Shapiro's syndrome: defining the clinical spectrum of the spontaneous paroxysmal hypothermia syndrome. Eur J Paediatr Neurol 2014;18:453-457. 